\title{
Modeling of an Electric Axle Drive with Modelica: A Study of Electric Active Dynamics
}

\author{
Hasan Flaih Awad \\ Frank Rettig \\ Tomas Smetana \\ Schaeffler Technology AG \& CO. KG \\ Industriestrasse 1-3, 91074 Herzogenaurach, Germany \\ \{awadhsa, rettifan, smetatma\}@ schaeffler.com
}

\begin{abstract}
This paper focuses on modeling of the electric axle drive system (eAxle) used for improving vehicle stability and handling performance by means of a torque vectoring (TV) feature as well as improving vehicle traction and reducing $\mathrm{CO} 2$ emissions by means of an electric traction feature. The function, construction and benefits of the eAxle will be explained within these contexts. An overview of the modeling of the eAxle in Dymola ${ }^{\circledR}$ will be shown. Several simulation cases are conducted to verify the effectiveness of the system for reducing fuel consumption and improving longitudinal and lateral dynamics. A co-simulation was developed between Dymola ${ }^{\circledR}$ and Abaqus ${ }^{\circledR}$ to simulate the power loss and ascertain the temperature behavior on the housing of the eAxle . Finally, the eAxle with a vehicle model was driven over a special realistic handling course using an open source software called Blender®. The dynamic behavior of the whole vehicle model (with eAxle) will be validated by means of an optical measurement process or what so called $(\mathrm{Ob}-$ ject Tracking).
\end{abstract}

Keywords: Electric axle drive system (eAxle); torque vectoring (TV); CO2 emission reduction.

\section{Introduction}

Nowadays, electric and hybrid vehicles are becoming increasingly important for many reasons, such as increasing oil prices and environmental pollution. Other contributory factors are legislation like, $\mathrm{CO} 2$ emission reduction, taxes and zero emission zones. Nonetheless, e-mobility vehicles are much more efficient than conventional vehicles because they can regenerate, store and reuse kinetic energy.

The Schaeffler Group develops three types of eAxle: A high-voltage (HV) eAxle, which is very suitable for electric and full-hybrid vehicles. This model has two electric motors (traction and TV). The second type is a 48-volt (48V) eAxle, which is optimally suited for mild- hybrid vehicles. It also has electric traction and TV motors. The third type is an $(48 \mathrm{~V}+\mathrm{TV})$ eAxle, which has just one $48 \mathrm{~V}$ electric motor that is used in traction mode until a predefined speed limit and it then uses as a TV motor. This type has 3 planetary gear stages, two for traction mode and the third for TV mode. The last type of eAxle is a huge step forward in the development of the eAxle. A HV eAxle [Figure 1] is presented in this paper.

The HV eAxle has a traction motor with a maximum power and torque of $65 \mathrm{~kW}$ and $160 \mathrm{Nm}$ respectively. With two planetary gear stages, the traction motor can drive the vehicle in full electric mode or assist the internal combustion engine (ICE).

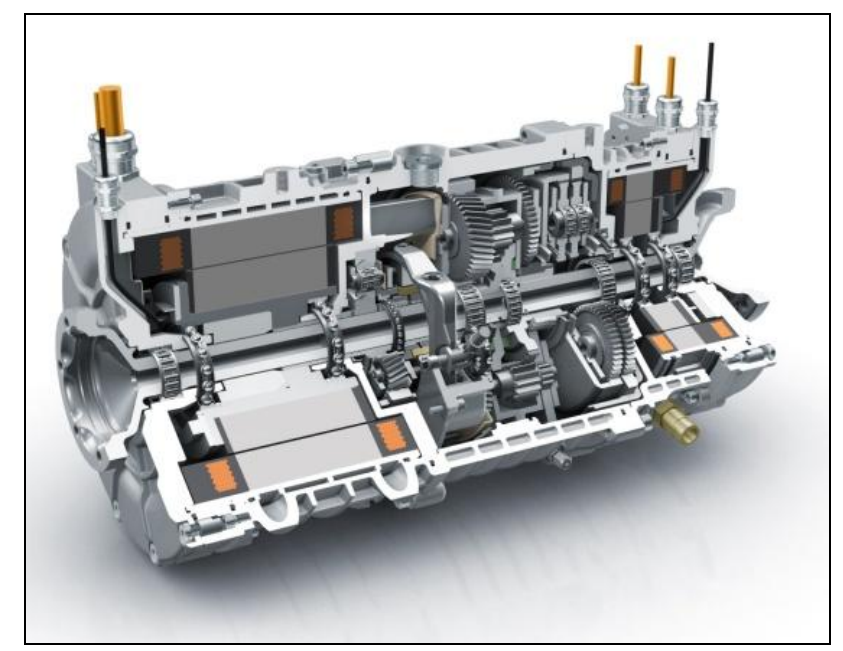

Figure 1: HV electric axle drive system

The TV motor has a maximum power of 6 $\mathrm{kW}$, which is transmitted via a planetary transmission to supply up to $1100 \mathrm{Nm}$ of delta torque between the left and right wheels.

The dynamics and agility of the vehicle can be significantly improved with the TV function. The steering characteristics are very responsive because 
there is less vibration and the driver has an improved feel of the road conditions through steering feedback. Traction is improved especially with regard to critical road conditions like $\mu$-split. The most important benefit of the TV function is the improvement to the stability and safety without any adverse effects on the longitudinal dynamics and traction. This means no power losses or velocity decrease in comparison with other stability technologies that use a brakesplit on different wheels.

An advantage of eAxle is the high potential for reducing fuel consumption and emissions because it is driven by electrical energy.

\section{Modeling of the eAxle}

The Schaeffler eAxle is a mechatronic system that contains mechanical parts (such as the transmission ), electrical parts (electric motors) and thermal and control systems. It therefore requires a modeling approach that allows modeling of multidomain systems. The modeling language Modelica ${ }^{\circledR}$ offers this facility and at the same time allows the model to be used on different simulation platforms. Therefore, the following model of the Schaeffler eAxle has been modeled using Modelica (www.modelica.org) in Dymola ${ }^{\circledR}$ which is a Modelica-platform tool.

\subsection{Transmission model}

The mechanics of the transmission [Figure 2] have been modeled with the $1 \mathrm{D}$ mechanics of the Modelica Standard Library (MSL). As an interface to the entire vehicle model, the transmission model has connectors for the torque vectoring motor, traction connector (which can be connected to the traction motor or ICE depending on the type of eAxle) and two output connectors (which are connected to the shafts of the left and right wheels). A deeper model level divides the model into the components "planetary gear sets", "differential" and "overlay gear set". The torsional rigidity of these components is determined by FEM analysis and mapped as linear springs in the model. The mass inertia is derived for all components from the CAD data and implemented as a rotating mass in the model.

The power losses in the traction path are implemented by means of a simulated map that has been calculated using the WTplus ${ }^{\circledR}$ program. This map is just a 3D matrix that depends on the torque, angular velocity and temperature (which will be calculated in Abaqus and imported back into Dymola by co-simulation).

The traction gear ratios are 12.34 for first gear and 4.22 for second gear. The TV gear ratio is -37.72 .

\section{2 eAxle model}

The transmission model is then integrated with the traction, TV motors and gearing signal into the eAxle model. The eAxle model has 3 input signals (throttle of the traction motor, which comes from the driver; throttle of the TV motor, which is regulated by the TV controller; and the required gear, which depends on the hybrid strategy).

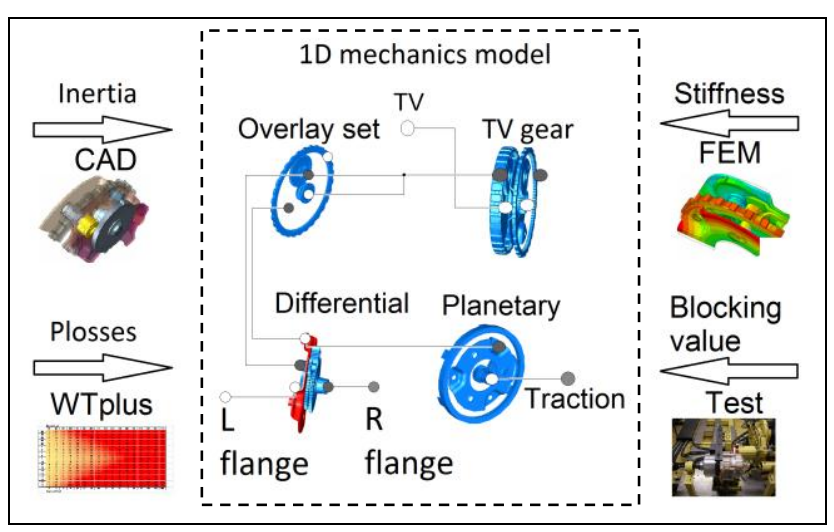

Figure 2: Transmission model

The efficiency-map and torque-over-angular velocity map of both motors (traction and TV) have been measured. The models of these motors have been implemented based on these maps (not a physical model)

The losses of the motors and transmission have been totalized to enable calculation of the actual temperature of the transmission and the temperature behavior on the eAxle housing by means of cosimulation with Abaqus.

The derating function of the electric motors has been defined by the motor supplier depending on thermal measurements of the motors. Thus, the derating function supplies a time-interval (for example, the traction motor is allowed to run with the maximum possible power for up to 5 seconds (overload time) and then has to run with less than or equal 
the nominal power for at least 20 seconds (relieftime)). This function has been implemented with help of StateGraph library of MSL as shown in [Figure 3].

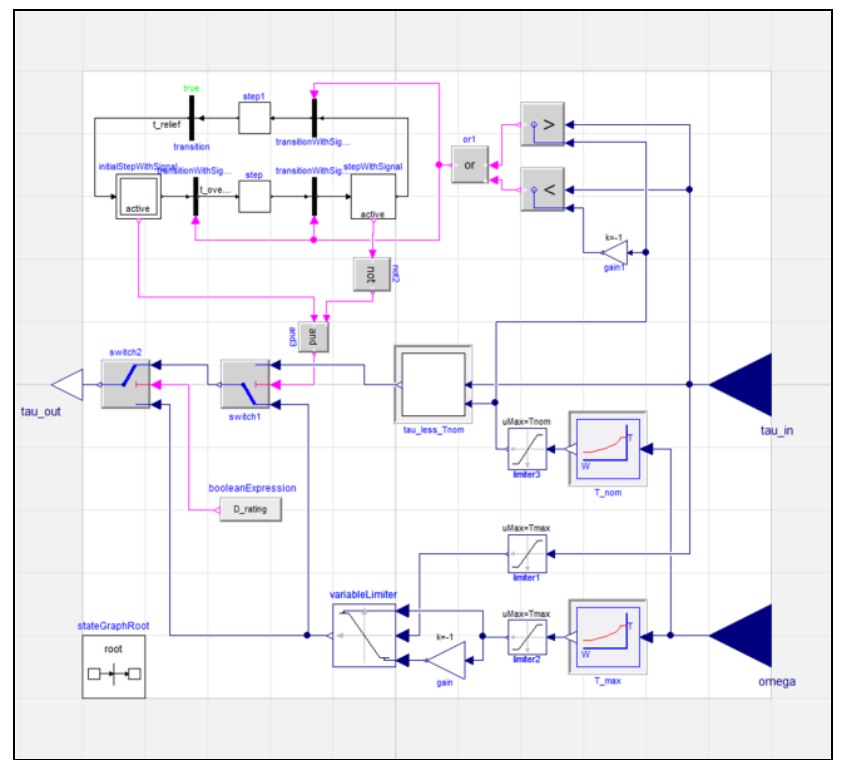

Figure 3: Derating model

\subsection{TV controller}

The TV controller is the unit responsible for defining the required torque of the TV motor in order to produce the necessary and effective yaw torque for the vehicle. [Figure 4] shows a diagram of the TV controller.

The TV controller calculates the desired yaw rate depending on the steering angle and some vehicle conditions as follows.

$$
\psi_{d}^{\bullet}=f\left(\delta, v_{x}, a_{y}, \beta\right)
$$

where, $\psi_{d}^{\bullet}$ is the required yaw rate, $\delta$ is the steering angle at the wheel, $v_{x}$ is the longitudinal velocity, $a_{y}$ is the lateral acceleration and $\beta$ is the side slip angle.

The required yaw torque is calculated by comparing the required and the actual yaw rate.

$$
T_{\text {yaw_d }}=f\left(\psi_{d}^{\bullet}, \psi_{\text {actual }}^{\bullet}\right)
$$

Finally, the required vectoring torques that should be supplied by the TV motors are calculated in the following equations.

$$
\begin{aligned}
& T v_{F A}=\alpha \cdot \frac{b \cdot R}{-2 \cdot n_{F}} \cdot T_{y a w_{-} d} \\
& T v_{R A}=(1-\alpha) \cdot \frac{b \cdot R}{-2 \cdot n_{R}} \cdot T_{y a w_{-} d}
\end{aligned}
$$

where, $T v_{F A}, T v_{R A}$ :Torque of TV motor of front/rear axle respectively, $\alpha$ : Fraction ratio of TV between front/rear axle (if $\alpha=0$ only rear axle TV is active, if $\alpha=1$ only front axle TV is active, otherwise the TV of both axles is active with that fraction ratio), $b$ is the vehicle track base, $R$ is the wheel radius and $n_{F}, n_{R}$ are the TV gear ratio of the front/rear axle respectively.

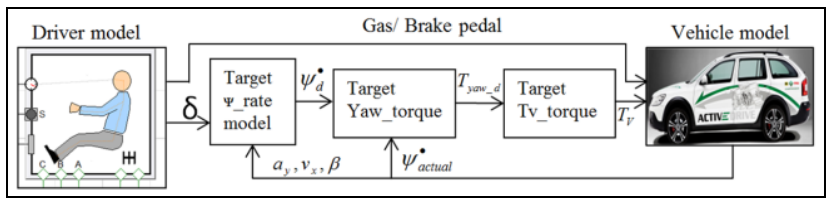

Figure 4:Diagram of TV controller

\subsection{Vehicle model}

The HV eAxle model was inserted into a drive train model and integrated together with a vehicle, driver and road models from the Vehicle Dynamics Library developed by Modelon®.

For the consumption analysis, the eAxle model was integrated with the rest of the vehicle model, driver and street models of the Power Train library developed by the DLR Institute of System Dynamics and Control.

\subsection{Co-simulation between Dymola and Abaqus}

A detailed thermal model of the eAxle has been modeled in Abaqus in order to carry out an adequate thermal analysis of the power losses in the eAxle due to the traction motor, TV motor and transmission. A co-simulation between Dymola and Abaqus is then used.

The Abaqus co-simulation technique can be used to solve complex systems that include electron- 
ics such as control systems, electro-mechanics, hydraulics, heat transfer and pneumatics by coupling Abaqus with Dymola, a general-purpose logical modeling software distributed by Dassault Systems. Structural responses computed by Abaqus/Standard or Abaqus/Explicit are coupled at run time with solutions provided by Dymola [6].

The Abaqus modeling method called "physical modeling" is the complementary modeling method to "logical modeling". Physical modeling is An FEM application, which uses a precise knowledge of the geometry to mimic the real world at a fine-grain level.

The Abaqus-Dymola coupled simulation capability targets simulations involving compliant response of a component or subassembly embedded in a larger, more complex system. All Abaqus features, including nonlinear materials, nonlinear geometric effects, and contact are available for use. In fact, any time-domain Abaqus model can be used as an Abaqus-Dymola co-simulation model with very few simple modifications[6].

The power losses of the eAxle in the Dymola model are defined with the same name as the actuators in the Abaqus model (Actuators are sources used to actuate the physical model). A virtual sensor is defined in the Abaqus model to measure the temperatures at some points and send them to Dymola. This sensor should have the same name as the input signal in the Dymola model. The Dymola model is then translated and the dymosim.dll file is selected by the plug-ins option in Abaqus.

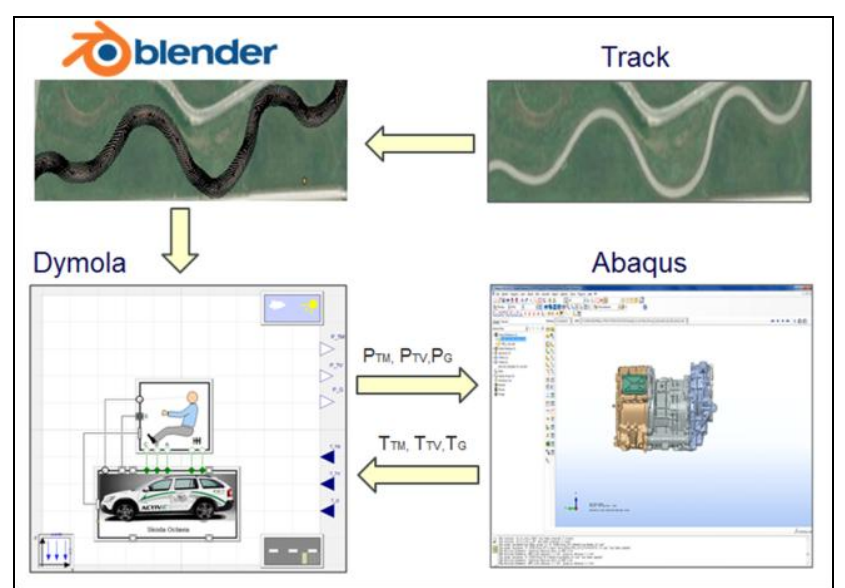

Figure 5: Co-simulation between Dymola and Abaqus, with a special realistic road.
In order to ensure a high level of actuation in the traction and TV motors, a special handling course road is used. The handling course road is imported as an image into Blender ${ }^{\circledR}$ in order to preprocess and generate the Modelica road file for implementation in the Dymola model [Figure 5]. The vehicle model is then simulated on the handling course road and the power losses of the eAxle are sent to Abaqus at every simulation step. Abaqus calculates the temperature values and sends them back to Dymola to run the next simulation step. These temperature values are necessary to calculate the power losses of the transmission.

\section{Simulation Results}

The consumption analysis and dynamic simulation of the vehicle have been calculated according to the vehicle data in Table 1 .

Some comparisons have been made between the conventional vehicle model (without an eAxle) and the hybrid vehicle model (with an eAxle). The hybrid vehicle model is $80 \mathrm{~kg}$ heavier than the conventional vehicle model (weight of eAxle) for all following simulation cases.

Table 1: Vehicle specifications

\begin{tabular}{|c|c|c|}
\hline \multicolumn{2}{|l|}{ Vehicle mass } & $2105 \mathrm{~kg}$ \\
\hline \multicolumn{2}{|c|}{ Dynamic tire radius } & $0.3186 \mathrm{~m}$ \\
\hline \multicolumn{2}{|c|}{ Frontal area } & $2.42 \mathrm{~m}^{\wedge} 2$ \\
\hline \multicolumn{2}{|c|}{ Friction coefficient of road } & 0.9 \\
\hline \multicolumn{2}{|c|}{ Moments of inertia of the vehicle } & $\begin{array}{l}\mathrm{Ixx}=709 \\
\mathrm{Iyy}=3281 \\
\mathrm{Izz}=3358 \\
\text { kg.m^2 }\end{array}$ \\
\hline \multicolumn{2}{|c|}{ Distance CG to the front, rear axle } & $1.269,1.308 \mathrm{~m}$ \\
\hline \multicolumn{2}{|c|}{ Vehicle track base } & $1.532 \mathrm{~m}$ \\
\hline \multicolumn{2}{|c|}{ Mass of eAxle } & $80 \mathrm{~kg}$ \\
\hline \multirow{3}{*}{$\begin{array}{c}\text { Traction } \\
\text { motor }\end{array}$} & Pmax & $65 \mathrm{~kW}$ \\
\hline & Tmax, Tnom & $160,140 \mathrm{Nm}$ \\
\hline & Speed max & $14000 \mathrm{rpm}$ \\
\hline \multirow{3}{*}{ TV motor } & Pmax & $6 \mathrm{~kW}$ \\
\hline & Tmax & $30 \mathrm{Nm}$ \\
\hline & Speed max & $14000 \mathrm{rpm}$ \\
\hline \multirow{3}{*}{ Battery } & Energy & $10 \mathrm{kWh}$ \\
\hline & $\mathrm{Vmax}, \mathrm{Vmin}$ & 365,267 Volt \\
\hline & SOCmax, SOCmin & $0.8,0.2$ \\
\hline
\end{tabular}

\subsection{Fuel consumption analysis}

To determine the save fuel by using the eAxle model, the vehicle model is driven in the New European Driving Cycle (NEDC) for $100 \mathrm{~km}$ with four different cases: Conventional, hybrid with a ful- 
ly charged battery, hybrid with a half charged battery and hybrid with a flat battery. The last case has been considered to specify the fuel saved in the case when the eAxle is used with no power in the battery, which means the eAxle can be driven only with the energy recuperated by braking. [Figure 6] shows the vehicle driving in two sequentially cycles of the NEDC. The eAxle firstly starts to recuperate the kinetic energy by braking and using it after that to drive the vehicle using electric power only for some period of time.

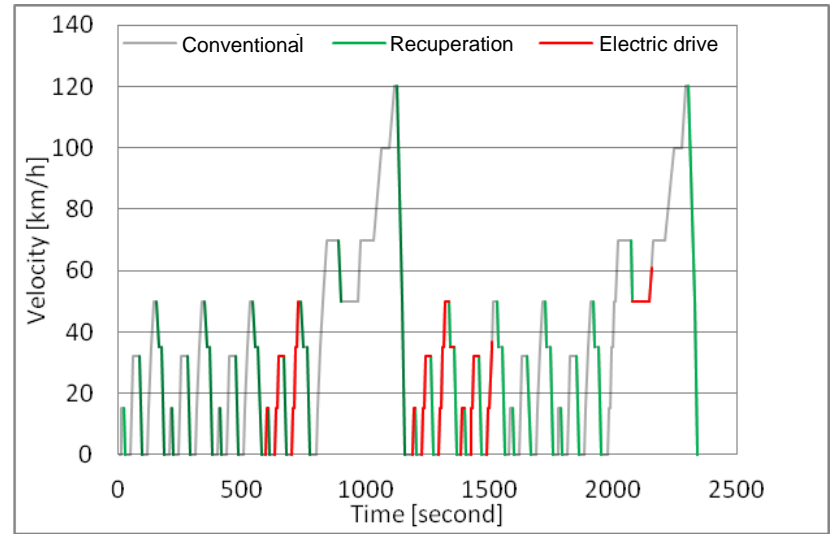

Figure 6: Hybrid vehicle driving in the NEDC with flat battery at beginning.

Although the eAxle makes the vehicle $80 \mathrm{~kg}$ heavier, it allows a reduction in fuel consumption of at least $18 \%$ compared with a conventional drive. If the battery is pre-charged, the eAxle enables a reduction in fuel consumption of up to $38 \%$ depending on the state of charge of the battery.

The fuel consumption over time and the percentage of fuel saved by the hybrid vehicle in comparison with a conventional vehicle when driving up to 100 $\mathrm{km}$ are shown in [Figure 7].

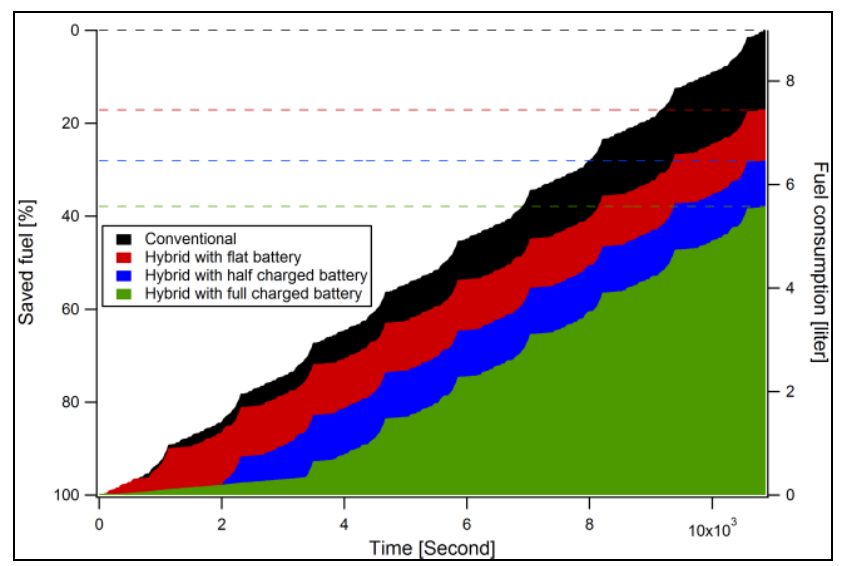

Figure 7: Fuel consumption in liters over time and percentage of overall fuel saving in a hybrid and conventional drive.

\subsection{Dynamic analysis}

\subsubsection{Longitudinal dynamic}

The eAxle almost makes a normal car into a sports car by using the $65 \mathrm{~kW}$ traction motor to assist the ICE.

Simulation results show that the vehicle model without an eAxle takes about 6.6 seconds to accelerate from 0 to $100 \mathrm{~km} / \mathrm{h}$, while the hybrid vehicle took about 5.1 seconds, which means the eAxle can improve the longitudinal acceleration of the vehicle by $29 \%$ on average as shown in [Figure 8].

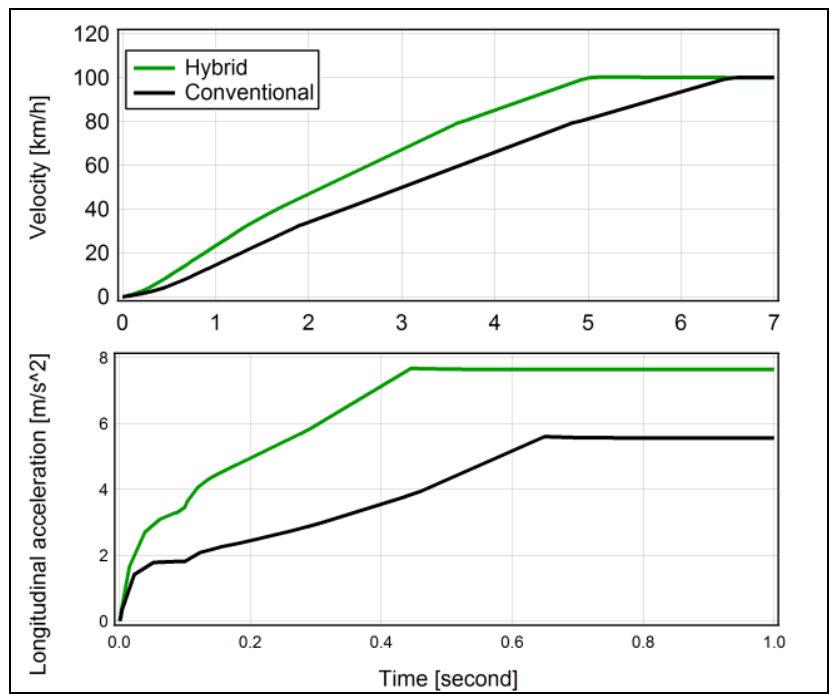

Figure 8: Acceleration from 0 to $100 \mathrm{~km} / \mathrm{h}$ for both a conventional and a hybrid vehicle. i) Vehicle velocities. ii) Longitudinal acceleration up to the $1^{\text {st }}$ second.

The eAxle can quickly supply a high traction torque even at low angular velocities, which gives the vehicle instantaneous high longitudinal acceleration. This fast response also gives the ICE a chance to supply a high torque in a shorter time because the eAxle drives the vehicle to a relatively high velocity which means the ICE runs with sufficient angular velocity to supply a high torque (ICE supplies low torques at low angular velocities). This explains the dramatic increase in longitudinal acceleration of the hybrid vehicle up to the $1^{\text {st }}$ second in comparison with a conventional vehicle, as shown in [Figure 8].

\subsubsection{Lateral dynamics}

The TV feature has a big influence on the lateral dynamics performance because the TV motor with a maximum torque of $30 \mathrm{Nm}$ can be used in conjunction with the TV gear to provide the vehicle 
with a torque difference of up to $1100 \mathrm{Nm}$ between the right and left wheels.

The vehicle is more stable and safer when it is driven in curves and can also be driven with a higher velocity. The driver will also notice the softer ride and ease of driving using the TV (driving pleasure).

Two simulation cases show the advantages of TV for the lateral dynamics, steering step response and cornering performance.

\subsubsection{Steering step response}

To verify the advantages of TV on the steering, the driver steers to 60 degree with 400 degree/sec while driving at $90 \mathrm{~km} / \mathrm{h}$. [Figure 9] shows the steering angle, while part $b$ shows the yaw rate of the vehicle with and without TV. It is so clear that the yaw rate of the vehicle has a smaller overshoot when using the TV because it increases the torque on the outer wheel and decreases torque on the inner wheel. The yaw rate also has less fluctuation and reaches a steady state faster because the TV compensates the fluctuation by biasing the difference in torque between the wheels.

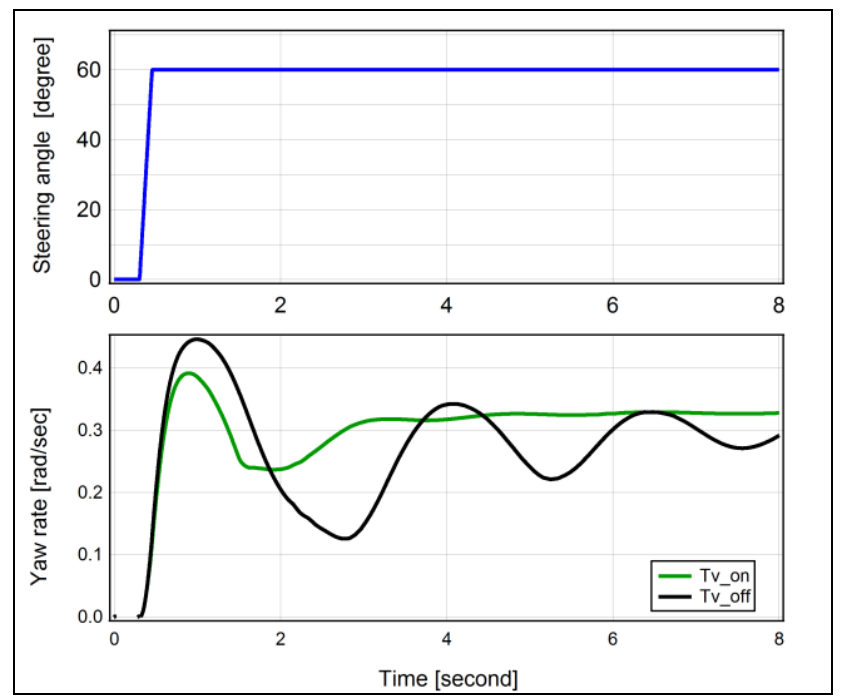

Figure 9: Step response test of a vehicle driven at 90 $\mathrm{km} / \mathrm{h}$. i) Steering angle. ii) Yaw rate with and without TV.

\subsubsection{Cornering performance}

A circular road with a $40 \mathrm{~m}$ radius curvature has been used to simulate the vehicle model with and without the TV function. The vehicle is driven at 65 $\mathrm{km} / \mathrm{h}$.

The simulation results [Figure 10] show that:

- TV decreases the gradient of the steering angle $(\delta)$ over the lateral acceleration of the vehicle [region1]. This means the steady- state behavior of the cornering performance of the vehicle is improved by using TV.

- The maximum level of lateral acceleration can be extended by using TV [region2], which gives the vehicle a wider range of stability.

- The TV decreases the necessary steering angle at high lateral acceleration, which is reflected in increased safety and driving pleasure.

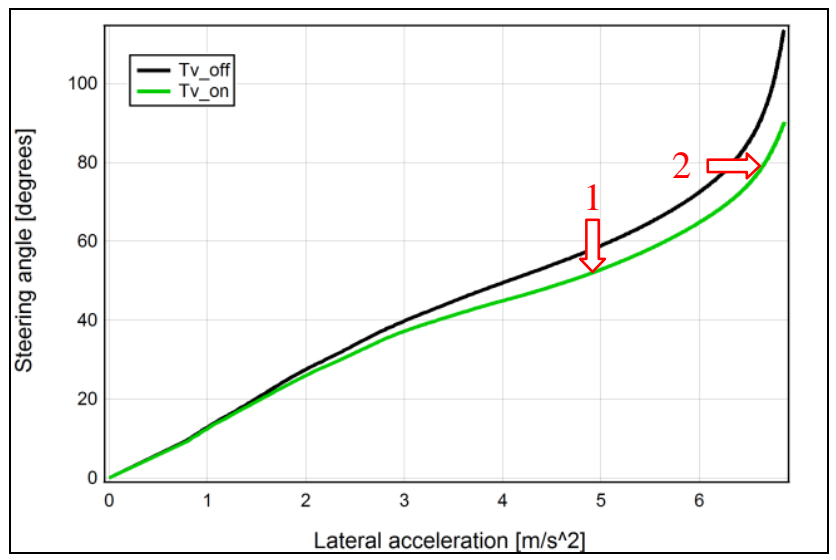

Figure 10: Steering angle over lateral acceleration with a $40 \mathrm{~m}$ radius curvature at $65 \mathrm{~km} / \mathrm{h}$.

The vehicle takes 8.2 seconds to negotiate the curve using TV and 8.3 seconds without TV. In addition, the total energy used by the traction and TV motor to negotiate the curve with TV is $1 \%$ less than the energy required when the TV is switched off (traction motor only) as shown in [Figure 11].

A reduction in fuel consumption of approximately $1.9 \%$ could be achieved by using the ICE instead of the traction motor of the eAxle (This configuration is suitable for special designs of lowvoltage eAxle). This means the TV feature not only improves the lateral dynamic performance, but also saves some fuel.

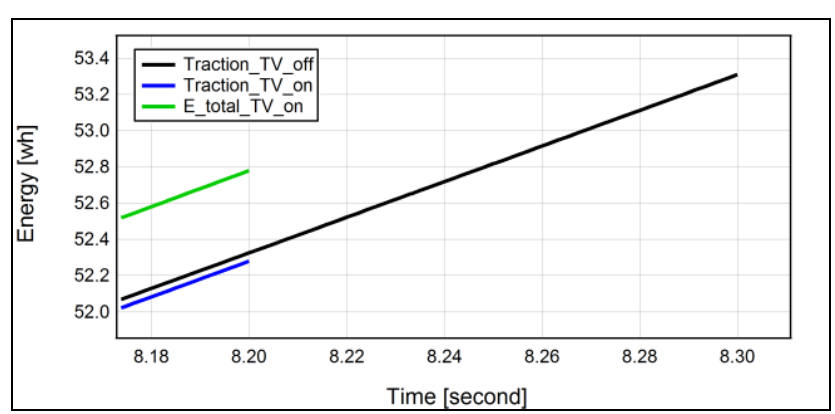

Figure 11: Energy consumption during cornering with and without TV. 


\subsection{Dymola-Abaqus co-simulation}

The vehicle is driven at $60 \mathrm{~km} / \mathrm{h}$ on the handling course road and Abaqus calculates the temperatures on the housing of eAxle depending on the power losses [Figure 12].

These temperatures are used to define the conditions of the electric or hybrid strategy.
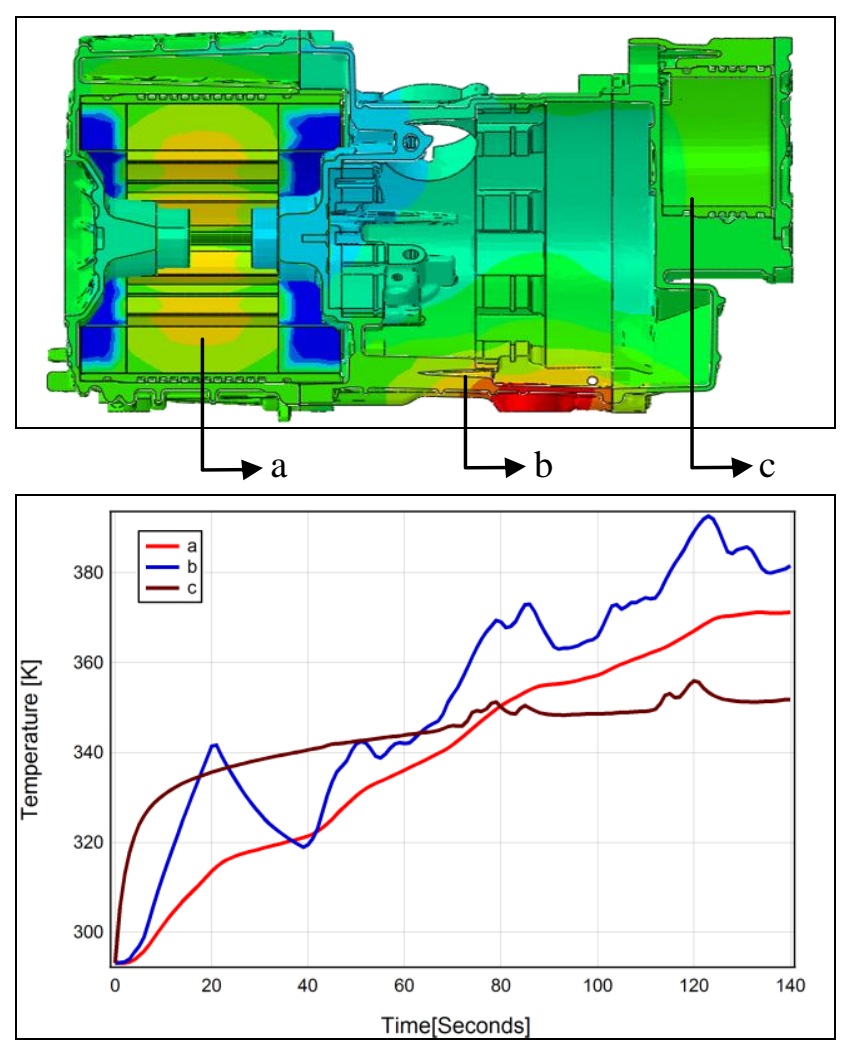

Figure 12: Temperatures on the eAxle housing a) on the traction motor. $b$ ) on the transmission and c) on the TV motor.

\subsection{Validation of vehicle model}

The entire vehicle model was validated on the basis of the lane-change test (ISO standard 38882) by using an optical measurement process. The measurement process analyzes the motion of optical markings [Figure 13.a] in a video recording of the driving maneuver (object tracking). With the corresponding software Blender ${ }^{\circledR}$, the variation in vehicle position and orientation on the test track can be determined over time[3]. A comparison between roll angle of the test vehicle by the object tracking and simulation result is presented in [Figure 13.b].

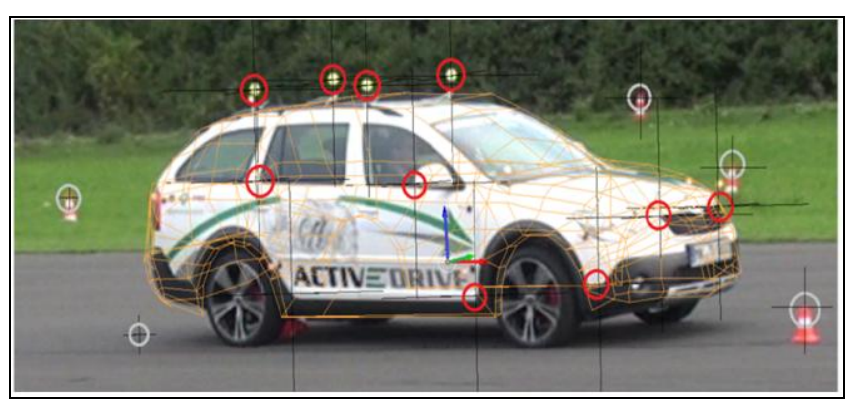

Inertial system. $\bigcirc$ Vehicle

a.

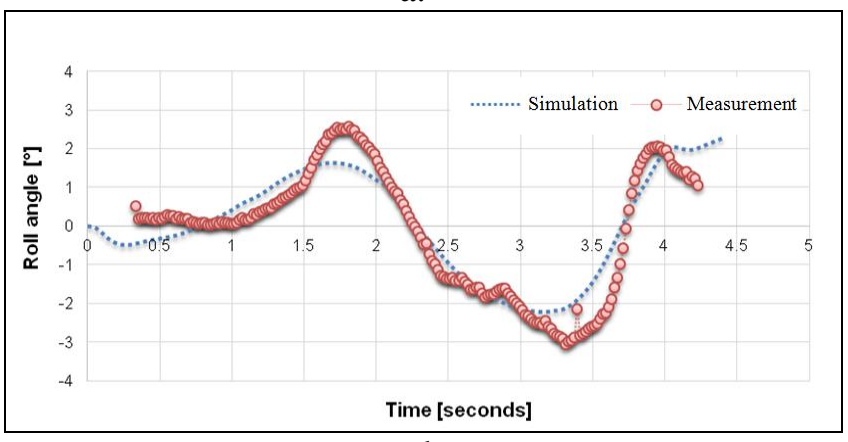

b.

Figure 13: a) Test vehicle with tracking markers. b) Roll angles in simulation and object-tracking validation.

\section{Conclusions and Outlook}

The modeling of a multi-domain system in Modelica, and the concept of building an electric active drive system for reducing fuel consumption , improving traction and providing yaw stability control have been discussed.

Modelica has a major advantage for dealing with a model that has many components from different disciplines.

Simulation results, which prove the advantages of the eAxle system in terms of dynamics and consumption are presented. A co-simulation has been developed between Dymola and Abaqus to investigate the thermal effects on the eAxle.

A realistic road has been constructed and preprocessed with other animation tools and can be easily and flexibly exported to Dymola .

Another simulation case has been started to investigate the effect of recuperation and the extra weight of the eAxle on the fuel consumption by considering the influence of elevation when the vehicle is driven on a road with a gradient. Unfortunately, the model of the driver in the PowerTrain library is unable to maintain the vehicle's velocity close to the 
requirement when the vehicle is driven downhill, which leads to non comparable consumption results.

Vehicle object tracking for validation the vehicle performance has been presented. A validated case between simulation result and reconstructed data from object tracking has been compared and shown.

\section{References}

[1] Smetana, „Schaeffler E-Axle Drive“.

[2] „Active electric differential for future drive trains“. Dr. Tomas Smetana, Thorsten Biermann, Prof.-Dr. Bernd-Robert Höhn, FZG München, Franz Kurth, FZG München and Dr. Christian Wirth, FZG München.

[3] „Lastdatenermittlung in Elektrischen Achsantrieben und Betriebsfestigkeitsbewertung für das Subsystem Elektrische-Maschine“.

D. Knetsch, F. Rettig, Dr. M. Funk, H. Awad, Dr. T. Smetana, M. Gramann. 40.Tagung des DVM-Arbeitskreises Betriebsfestigkeit.

[4] „Das aktive eDifferenzial für den Antrieb der Zukunft".

13. ITI-Symposium. Frank Rettig, Dr. Tomas Smetana and Roman Sauer.

[5] „Interfacing Abaqus with Dymola: A High Fidelity Anti-Lock Brake System Simulation". Modelon AB , $7^{\text {th }}$ Modelica Conference 2009.

[6] „Logical-Physical Modeling Using Abaqus and Dymola". Dassault Systems, 2012

[7] Modelica ${ }^{\circledR}$ - A Unified Object-Oriented Language for Systems Modeling. Language Specification. Version 3.3.

[8] Modelica ${ }^{\circledR}$, http://www. Modelica.com.

[9] Abaqus®, Dassault systems http://www.3ds.com.

[10] Blender 3D. http://www.blender.org. 